Documento No. 13

\title{
Intermediarios Financieros y \\ Mercados Imperfectos de Capital
}

por

Guillermo Ortíz

Septiembre, 1979

Las ideas contenidas en le presente ensayo son responsabilidad exclusiva del autor y no reflejan la posición del Banco de México, S.A. 
Intermediarios Financieros
Mercados Imperfectos De Capital

Por

\section{Guillermo Ortíz}

\section{INTRODUCCION}

Este documento dos divide en dos partes. En la primera parte se examinan los principales aspectos teóricos del papel que la intermediación financiera representa en el desarrollo económico, y se arguye que la intermediación financiera es el resultado de ciertas características de la estructura competitiva del mercado de capital. Esto nos lleva a discutir la función de asignación de los mercados de capital en la hipótesis de que funciona eficientemente. En virtud de que nuestro interés principal estriba en las características de economías en desarrollo, este ensayo se concentra más adelante en situaciones donde los mercados de capital no son perfectos.

En los últimos años, especialmente a raíz de la divulgación del trabajo seminal de Gurley y Shaw(6), se ha producido un volumen creciente de literatura en materia de finanzas y desarrollo. No obstante, en el año de 1969, Goldsmith escribió: "“Para evaluar el papel del desarrollo y la estructura financiera en el crecimiento económico podríamos dirigirnos, según nuestras predilecciones filosóficas, hacia la teoría económica o hacia la historia de la economía. Desafortunadamente, nos encontraremos con que en el estado actual de la teoría del crecimiento económico, y con la escasez presente de estudios históricos suficientemente exhaustivos sobre desarrollo financiero, no podemos obtener respuestas precisas de cualquiera de las dos disciplinas" (7 p. 391). Esta situación ha mejorado desde entonces. Los nuevos trabajos realizados por McKinnon y Shaw han contribuido con una base teórica substancial a la noción, durante mucho tiempo aceptada, del papel potencialmente importante que las finanzas representan para el crecimiento económico (13 y 20). La primera parte de este documento se destinará a los temas teóricos abordados por dicho trabajo.

Dada la importancia de las imperfecciones de los mercados de capital para entender el papel de la intermediación financiera, la segunda parte del trabajo se concentra en un examen de los tratamientos teóricos sobre mercados de capitales imperfectos aparecidos en la literatura económica reciente. Aquí se abordan aspectos de las decisiones de agentes económicos, empresas y consumidores, cuando se

\footnotetext{
* Documento preparado originalmente en inglés. Traducido por la Oficina de Traducciones del Banco de México, S.A.
} 
enfrentan a situaciones específicas de imperfecciones en el mercado de capitales, tanto bajo condiciones de certidumbre como bajo incertidumbre. También se incluye en esta sección una discusión detallada del desarrollo histórico de los conceptos de riesgo e incertidumbre, desde el trabajo de Khight hasta el enfoque moderno de "estados de la naturaleza” de Arrow y Debreu.

\section{Economías "Monetizadas" e Intermediarios Financieros}

\subsection{Intermediación y Crecimiento}

¿De qué manera puede influir la intermediación financiera en le crecimiento económico?. Existen dos mecanismos viables: uno es mediante el incremento de rendimiento de la inversión elevando el producto marginal (o medio) de los factores de producción empleados, y el segundo es a través del aumento del volumen total de fondos destinados a la inversión. En el contexto de un modelo de crecimiento neoclásico, esto implica un desplazamiento hacia fuera de la función producción o una relación capital/mano de obra equilibrio mayor.

La justificación teórica de la existencia de una superestructura financiera, y de la presunción de que acelera el crecimiento económico, se expresa generalmente en forma similar al argumento relacionado con las ventajas del cambio indirecto sobre el trueque. Es decir, que libera a los factores de producción de las actividades de cambio mediante la reducción de los costos de las transacciones. La creación de instrumentos financieros libera a los agentes económicos del vínculo que existe entre sus ahorros y su inversión. Una vez que se introducen los instrumentos financieros, las unidades ya no están limitadas al autofinanciamiento y pueden invertir o consumir más allá de la frontera de sus posibilidades de producción.

Este resultado de "separación" (de la existencia de instrumentos financieros) da por sentado implícitamente que las oportunidades de inversión y las habilidades empresariales son diferentes entre la población. Se define a los servicios financieros como el proceso mediante el cual se hace posible la transferencia de recursos de las unidades económicas superavitarias a las unidades deficitarias que invierten estos recursos. Si ambas tuvieran la misma tasa de rentabilidad, nada se ganaría con esta transferencia. Existe otro factor que, aún en la ausencia de oportunidades de inversión desiguales puede convertir a la introducción de instrumentos financieros en potencialmente productiva: la

\footnotetext{
${ }^{1}$ Véase por ejemplo Goldsmith (7).
} 
existencia de indivisibilidades. Si la escala mínima de eficiencia de un proyecto de inversión es tan extensa que ningún individuo particular puede emprenderlo, el solo acto de mancomunar recursos por conducto de instrumentos financieros podría, de hecho, tener un efecto positivo sobre el crecimiento. ${ }^{2}$

Algunos autores han intentado formalizar la relación existente entre la estructura de las finanzas y el nivel de producción y tecnología. En particular, Spellman (17 y 21), siguiendo la formulación de Shaw, asocia la estructura financiera con la frontera de posibilidad de producción: el impacto de cambios financieros se puede reflejar en un desplazamiento de dicha frontera (debido a las variaciones en la disponibilidad total de factores) o en un movimiento a lo largo de la misma (debido al incremento en el rendimiento de la utilización de factores originados por la existencia de un sistema financiero). En este contexto, los efectos de las finanzas en el crecimiento económico pueden ser analizados coherentemente y separados adecuadamente. A continuación presenta una formulación alternativa.

Imaginemos, para simplificar, una economía que produce un solo bien $\mathrm{Q}$, con dos insumos $\mathrm{K}, \mathrm{L}$ y una tecnología financiera dada $\mathrm{T}$ que se puede representar como:

$$
Q=F(K, L ; T)
$$

Se supone que las segundas derivadas parciales de $\mathrm{F}$ son continuas con respecto a las tres variables, y para cualquier $\mathrm{T}_{0}$ dado, $\mathrm{F}\left(\mathrm{K}, \mathrm{L} ; \mathrm{T}_{0}\right)$ tiene las propiedades neoclásicas habituales. Una forma de expresar el "efecto de separación" de las finanzas que se mencionó anteriormente sería volver a representar (1) como una función de producción en la que los factores K y L se incrementan

$$
Q=F(K, L ; T)=G[\alpha(T) K, \beta(T) L]
$$

En un mundo en el que el costo de las finanzas en términos reales es cero, el punto de vista adoptado por la mayoría de los autores de tradición neoclásica, prevalecería una relación tal como la (2) ${ }^{3}$. Si el suministro de servicios financieros requiere los insumos de ambos factores $\mathrm{K}_{\mathrm{f}}, \mathrm{L}_{\mathrm{f}}$, que representan un costo en función del producto sacrificado, podemos expresar la función de producción como

$$
Q=G[\alpha(T) K, \beta(T) L]-C_{T}\left(K_{f}, L_{f}\right)
$$

\footnotetext{
${ }^{2}$ Ibid. Cap. IX.

${ }^{3}$ Ver E. Shaw (20) Cap. 2.
} 
Si G y C son homogéneas de grado uno, obtenemos

$$
q=g(z)-c_{T}(k)
$$

donde $g(z)=G(z, 1)=G\left[\frac{\alpha(T) K}{\beta(T) L}\right]=\frac{Q}{\beta(T) L} \quad$ y $\quad k=\frac{K_{f}}{L_{f}}$

Esta representación se ilustra en la Gráfica 1.

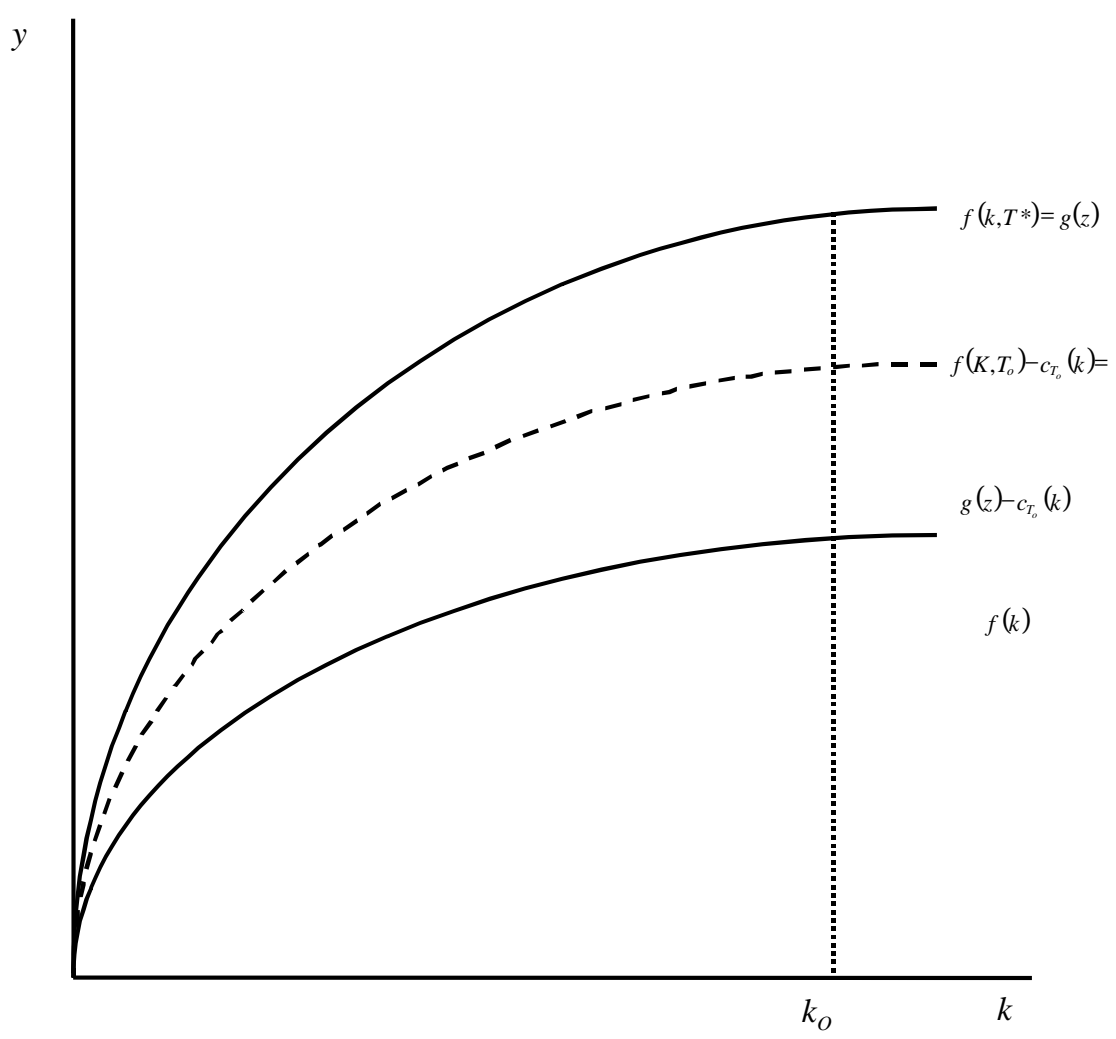

Para un nivel determinado de $k$, digamos $k_{0}$, la producción per cápita está limitada en la parte inferior por la función de producción de la economía de trueque, $f(k)$. El límite superior lo constituye la función de la producción de una economía totalmente monetizada, $f(k, T *)$, en la cual las finanzas no tienen costo. Entre estos límites se encuentran las funciones de producción de economías con diferentes tecnologías financieras que utilizan recursos reales representados por la relación costo $C_{T_{0}}$. 
Ahora es fácil representar el impacto de las finanzas y de la innovación financiera en la economía (para cada nivel de k) como la transición de $f(k)$ a $f(k, T *)$. El límite superior que corresponde a la economía totalmente monetizada, compatible con la tradición literaria que el profesor Shaw define como "el punto de vista de la riqueza" (wealth view) sobre el dinero y las finanzas ${ }^{4}$. En esta economía, ninguna variación en las técnicas para crear, retener y cambiar valores puede aumentar la cantidad demandada de los mismos para cada tasa de rendimiento. Es un mundo de mercados de capital perfectos (que se definirá con más precisión a continuación) si no existe incertidumbre, y de mercados capitales completos (en le sentido de Arrow-Debrreu) si hay incertidumbre. El grado de sofisticación de las técnicas financieras ha eliminado toda incertidumbre en relación con las oportunidades de intercambio de manera que participan en le proceso de intercambio no incurrirán en "costos de búsqueda". Todas las imperfecciones del mercado de capital han sido eliminadas en esta etapa, la información se distribuye uniformemente y se obtiene sin costo alguno, de modo que en esta economía no se necesitan intermediarios financieros.

Cuando la economía alcance el límite superior, ya habrá completado un círculo. El primer paso hacia la "monetización completa", empezando desde una situación de trueque, es la introducción del dinero respaldado por productos o bienes: un numerario. Más tarde, hacen su aparición la moneda no respaldada y el financiamiento directo; el financiamiento indirecto (la intermediación), se presenta probablemente en una etapa posterior. Pero cuando la economía se aproxima a esta situación ideal, las imperfecciones del mercado de capital que hicieron útil el papel de intermediación, empiezan a desaparecer gradualmente obligando así a la economía a regresar al financiamiento directo. Además, ya no se necesita el dinero en este tipo de economía. Se pueden realizar transacciones en le mercado por cualquier monto.

Hasta este momento, el papel del financiamiento indirecto y de los intermediarios financieros no ha sido tratado de manera explícita. Consideremos una ilustración inspirada en Hicks (8,cap 13).

\subsection{Intermediarios Financieros.}

Pensemos en una economía compuesta por ahorradores, empresas y un solo banco. Supongamos que los agentes mantienen cuatro clases de activos: dinero (M), acciones de empresas (E), crédito de bancario (L) y capital físico (K).

\footnotetext{
${ }^{4}$ Ibid. Cap. 2.
} 
Con cada activo esta asociada una tasa de rendimiento $r_{m}, r_{e}, r_{l} \mathrm{y} r_{k}$. Los activos están distribuidos de la siguiente forma:
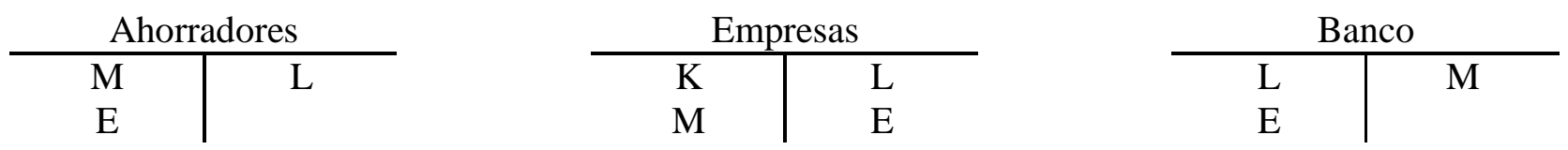

La cuestión es, entonces examinar la relación que existe entre los rendimientos de los diferentes activos. Si las tasas no son iguales, ¿por qué difieren?. ¿Cómo es posible que un individuo pueda retener simultáneamente activos con diferentes rendimientos?. La respuesta se obtiene en la teoría de la selección de cartera cuando existe incertidumbre, la cual a su vez se deriva de la noción Keynesiana de la preferencia de la liquidez

Partiendo de una situación de equilibrio, un cambio en la composición de los activos de los ahorradores de dinero por acciones implica una pérdida de liquidez; por lo tanto, exigirán una mayor rendimiento de la empresa que del banco ${ }^{5}$. Si las empresas desean ampliar sus tendencias de activos reales y contratan créditos con el banco o venden acciones al público con este fin, su liquidez también decrecerá. En consecuencia, se mostrarán renuentes a pagar intereses más elevados sobre L ó E de los que esperan recibir. Esto implica $r_{m}<r_{l}, r_{e}$. El banco, por otro lado, no tiene problema de liquidez real, ya que sus pasivos son los valores más líquidos que existen. Siendo que el banco puede crear sus propios pasivos emitiendo préstamos, se podría pensar que, en ausencia de una autoridad monetaria central, se llegaría a una continua expansión monetaria. No obstante, es bien sabido que este no es el caso $^{6}$. La demanda de dinero en esta economía depende de su tasa de rendimiento y la de otros activos con los que compite. Desde el punto de vista del banco, sus préstamos se verán restringidos por los beneficios y riesgos potenciales. Si el banco quiere continuar operando, los rendimientos marginales de los préstamos al público y de las inversiones en otros activos que superan el costo marginal de la captación y la retención de nuevos pasivos. Esto implica que $r_{m}<r_{l}$ y que la oferta de medio circulante no es perfectamente elástica ${ }^{7}$. Hasta aquí tenemos las siguientes relaciones:

$$
r_{e}>r_{m} \quad r_{k}>r_{l}, r_{e} \quad r_{e}>r_{m}
$$

\footnotetext{
${ }^{5}$ Por lo tanto, $r_{e}>r_{m}$

${ }^{6}$ Ver, por ejemplo, J. Tobin (24).
} 
que en conjunto implican:

$$
r_{m}<r_{m}>r_{e}<r_{k}
$$

Existe un límite máximo a todas las tasas de interés que es la tasa de rendimiento sobre inversiones en términos reales, y uno mínimo que fija la tasa de interés pagado por el banco. Las demás tasas de interés deben de mantenerse dentro de estos límites.

Podría caber en este ejemplo un intermediario financiero si se toma en cuenta el límite de expansión crediticia. En esta economía, el crédito esta limitado:

a) Por las consideraciones de liquidez de parte de las empresas prestatarias.

b) Por la existencia de incertidumbre del banco con respecto a las perspectivas de inversiones en términos reales que desean emprender las empresas, que plantea finalmente la posibilidad de incumplimiento.

Un intermediario financiero puede prosperar si logra transformar el carácter de la deuda del prestatario y la naturaleza del activo del último prestamista, con el fin de manejar en una forma más eficaz los costos de transacción. Esto implica utilizar (o elaborar) información especializada sobre las perspectivas de las empresas particulares que no están a disposición del banco, o cuya elaboración resulta muy costosa para el mismo. Por razones similares a las presentadas en el caso del banco, la tasa a la que el intermediario absorbe fondos tiene que ser menor a la que cobra por la venta de sus activos $r_{m_{2}}<r_{m_{2}} l$. En esta forma, podemos desarrollar toda una jerarquía de instituciones especializadas que emiten valores cuyas tasas de rendimiento tienen que comprimirse entre el nivel mínimo ${ }^{r_{m}}{ }^{1}$, y el máximo, ${ }^{1}{ }^{1}$.

Suponiendo que $r_{k}$ esta determinando en le parte "real" de la economía (una tasa de interés natural de tipo Wicselliana), debe de existir una tasa básica pagadera por le banco sobre depósitos que dejará espacio a una estructura de tasas de interés consistente con un equilibrio monetario.

\footnotetext{
${ }^{7}$ Habla técnicamente, un monopolista (el banco en este caso) no tiene una curva de oferta. Es posible construir una, sin embargo, el desplazando la demanda.
} 
El tamaño de la brecha existente entre la tasa de rendimiento del capital $r_{k}$ y la tasa pagadera por depósitos monetarios $r_{m}$, se determina por la piramidación de los márgenes entre tasas "de depósito" y "de préstamo" de las instituciones financieras. Uno de lo efectos de la introducción de intermediarios financieros es precisamente de reducir esta brecha. ¿Cómo se logra esto?. Los intermediarios ofrecen a los prestatarios (que desean ampliar sus inversiones en activos reales) tasas más bajas y términos más cómodos de lo que hubieran recibido de haber recurrido directamente al público, o a los prestamistas no especializados. A su vez, si los acreedores de instituciones financieras tuvieran que adquirir directamente las obligaciones emitidas por prestatarios privados, insistirían en establecer tasas más altas y términos más estrictos.

La proliferación de intermediarios especializados tiende a reducir los costos de operación de la economía. Al intensificar la competencia por fondos, los intermediarios financieros promueven una mayor eficiencia en la utilización de recursos reales involucrados en la producción de servicios financieros. En términos de la formulación anterior, dichas instituciones reducen al mínimo la función $C_{T}\left(K_{p}, L_{f}\right)$. Suponiendo que cada intermediario sólo emite una obligación e indicando con la letra $\mathrm{N}$ el número de dichos instrumentos, es posible expresar la brecha entre las tasas de interés como una función inversa del número de instituciones:

$$
r_{k}-r_{m}=f(N)
$$

de manera que

$$
\lim _{N \rightarrow \infty}\left(r_{k}-r_{m}\right)=0
$$

abstrayendo los costos administrativos.

Lo que constituye el papel fundamental de la intermediación es la disminución de la dispersión en la estructura de las tasas de interés en un momento dado. Consecuentemente, las discrepancias entre las tasas de rendimiento de los activos reales tenderá también a reducirse, disminuyendo también la segmentación del mercado de capitales. Este efecto de distribución ha motivado a McKinnon (13) a definir el desarrollo económico como "la reducción de la gran dispersión de tasas sociales del rendimiento de las inversiones existentes y de los nuevos proyectos que se encuentran bajo control empresarial doméstico". 


\section{EL Mercado de Capital y las Decisiones de Inversión.}

\subsection{Decisiones en Ausencia de Incertidumbre.}

Se ha indicado que en una condición necesaria para la existencia de instrumentos financieros indirectos y de dinero, es que el mercado de capital sea imperfecto en algún sentido. En el caso del dinero, en ausencia de imperfecciones en el mercado de capital, su existencia a nivel teórico tiene que ser justificada suponiendo que la tenencia de dinero entra directamente en las funciones de utilidad. Sin embargo, parece que este concepto está en conflicto con el marco teórico de "selección" de la economía neoclásica que postula que la utilidad produce únicamente el consumo y no simplemente la tenencia de activos. No obstante, la mayor parte de la literatura relacionada con la economía monetaria, y la teoría financiera que se deriva de fundamentos microeconómicos, supone la existencia de mercados de capital perfectos. De aquí que no resulte sorprendente el hecho de que el papel que juega la intermediación financiera permaneciera inexplorado durante tanto tiempo en la literatura.

Gurley y Shaw explicaron (con razón) que el dinero, siendo un activo financiero, debería de ser estudiado en una teoría más general sobre finanzas que incluyese a la teoría más general sobre finanzas que incluyese a la teoría monetaria. Los activos financieros forman por sí mismos parte de una categoría más amplia: activos en general o depósitos de valor. Como tales, pertenecen a la teoría del capital. Por tanto, resulta necesario examinar más de cerca los mercados de capital, si se quiere comprender el impacto de la intermediación financiera en el crecimiento económico. También es evidente que la hipótesis de mercados perfectos es una mala aproximación a la situación prevaleciente en los países en vías de desarrollo.

Por estas razones se hace necesario estudiar los mercados de capital imperfectos. Examinamos aquí las decisiones que toman individuos y empresas en algunos casos relevantes de imperfecciones en el mercado de capital. Es sorprendente que se haya escrito tan poco acerca de este tipo de mercados; una de las razones puede ser que tratándose de mercados imperfectos en general, no se logran obtener resultados "elegantes" de equilibrio general. El argumento que se presenta a continuación se llevará a cabo en un mundo Fisheriano de dos períodos, pero antes, conviene brevemente aclarar el concepto de mercados perfectos e imperfectos.

El mercado de capital es la unión que existe entre la economía presente y futura. Ofrece los medios para que las unidades económicas individuales intercambien recursos y productos que estarán disponibles en diferentes momentos de tiempo; además, libera a los individuos de sus propios límites 
de posibilidades de producción y les permite distribuir sus decisiones de consumo conforme s sus preferencias en el tiempo. El mercado de capital juega un papel central tanto en la teoría monetaria como en la teoría financiera.

Sólo existe un producto final que se comercia en los mercados de capital: los flujos de ingresos. Estos flujos pueden "incorporarse" en distintos activos cuyos precios relativos se determinan competitivamente. En general, los de capital serán perfectos si reúnen los siguientes requisitos ${ }^{8}$ :

1. Todos los participantes tienen acceso sin costo a la información sobre precios corrientes y las otras propiedades relevantes de los artículos que se comercian (valores).

2. Todos los participantes se enfrentan a precios dados (son "price takers"). Por consiguiente, cada uno se comporta como si su actividad dentro del mercado no tuviera efecto alguno sobre los precios corrientes.

3. No existen honorarios de corretaje, impuestos de transferencia u otros costos de operación en lo que se incurra en el momento en que se compran, venden o emiten valores.

\subsubsection{Mercados Perfectos}

Pensemos en le tradicional paradigma Fisheriano de dos períodos en el que existen mercados perfectos y no hay incertidumbre ${ }^{9}$. Cada individuo está dotado con unidades de bienes de consumo presente y con un conjunto convexo de oportunidades de producción. Sólo existe un producto que se puede utilizar para el consumo actual; se puede vender en le mercado de capital o utilizar como un insumo para la producción de unidades de productos de entrega futura. Si el mercado de capital es perfectamente competitivo, como se describió anteriormente, entonces la decisión de producción óptima para el consumidor (o la empresa) es la de maximizar el valor de mercado de la mercancía producida. Esto se obtiene en el punto de intersección entre el conjunto de oportunidades de producción y la línea de intercambio del mercado de capital más lejana del origen y tangente al conjunto de oportunidades. Se ilustra en la Gráfica 2 donde $\bar{C}\left(\overline{C_{0}}, \overline{C_{1}}\right)$ corresponde a la dotación en

\footnotetext{
${ }^{8}$ Ver, por ejemplo, Fama y Miller (5), Cap. 4.

${ }^{9}$ Hirshleifer (9) presenta una excelente exposición del modelo.
} 
función de los productos en los períodos uno y dos. $P^{*}\left(P^{*}{ }_{0}, P^{*}{ }_{1}\right)$ es la selección de la producción óptima y $C^{*}\left(C^{*}, C^{*}{ }_{1}^{*}\right)$ la decisión de consumo óptimo.

\section{Gráfica 2}

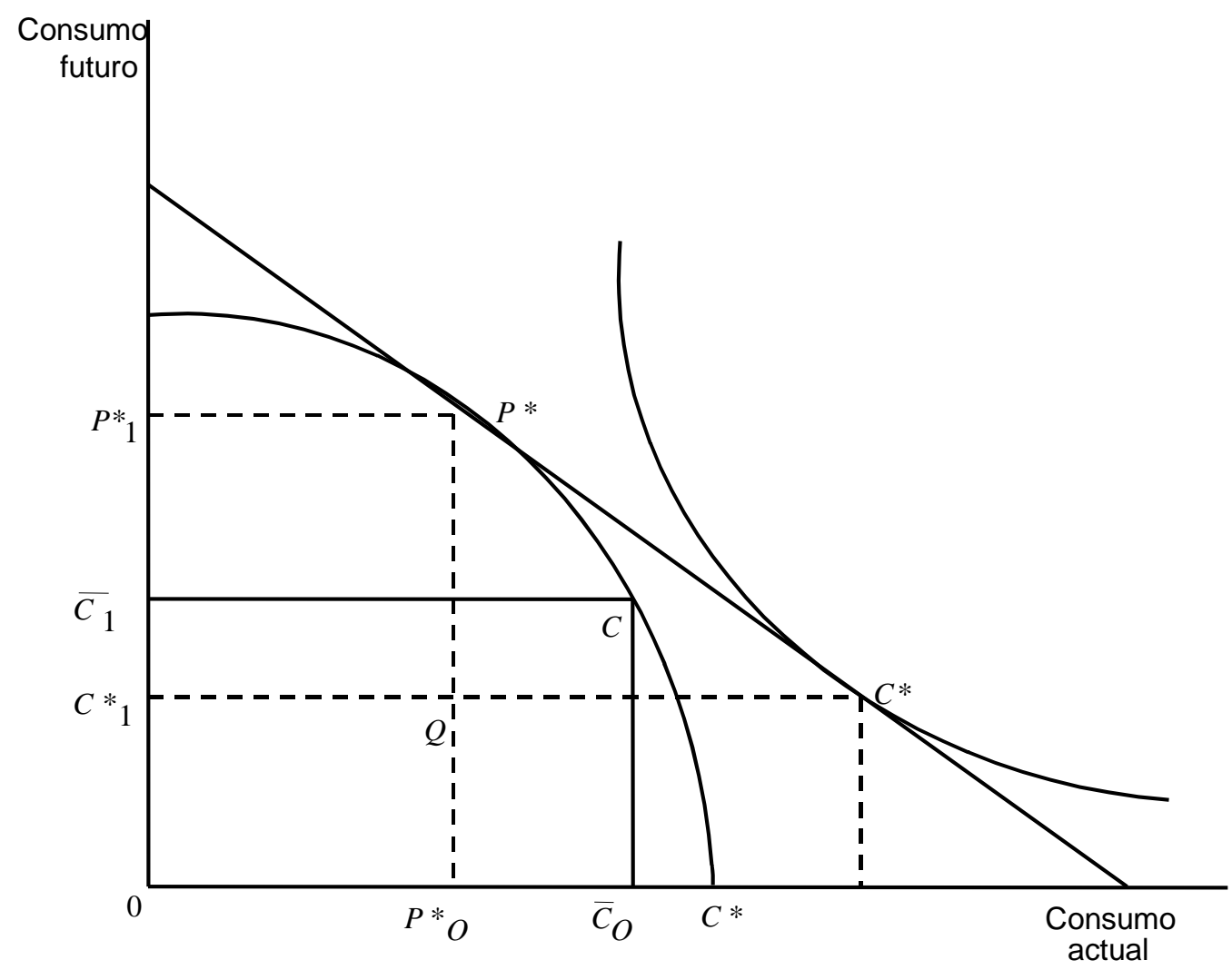

Originalmente el individuo está dotado con $\overline{C_{0}}$ unidades de $C_{0}$ y $\overline{C_{1}}$ unidades de $C_{1}$. Conociendo su propia tecnología y sus gustos, la única información adicional que necesita el inversionista es el vector de precios. Conocido éste, el individuo invertiría $C_{0}-P_{0}^{*}$ y produciría en le punto $P^{*}$. Esta decisión es independiente de las preferencias del individuo, ya que éste puede consumir lo que desee a lo largo de la línea de intercambio del mercado de capital. En este caso, el individuo pide prestado $Q C^{*}$ a la tasa de $r=\frac{P^{*} Q}{Q C^{*}}$ y que consume $C^{*}{ }_{0}$. En el siguiente período reintegra $C{ }^{*}{ }_{1} P^{*}{ }_{1}$ de la producción total, y el consume el remanente de la producción $O C{ }^{*}$. Este 
principio de separación es uno de los resultados más importantes de la teoría de las finanzas. Un segundo resultado básico(conocido como el Teorema1 de Modigliani-Miller) es que, dada su decisión de producción-inversión, el valor de una empresa en el mercado en cualquier momento se determina de manera independiente de sus decisiones de financiamiento; es por ello que las decisiones de operación no tienen por qué ser afectadas por las decisiones de financiamiento. Este principio se discutirá más adelante.

\subsubsection{Mercados Imperfectos: Costos Crecientes de Endeudamiento}

Continuando en el esquema Fisheriano de los períodos, los ingresos del segundo período, $R_{2}$, pueden expresarse como una función explícita de las inversiones realizadas en el primer período, $I_{1}$ :

$$
R_{2}=f\left(I_{1}\right)
$$

A su vez, la tasa interna de rendimiento marginal puede definirse como

$$
\rho=\frac{\partial R_{2}}{\partial I_{1}}-1=f^{\prime}-1
$$

entonces, la función objetivo de la empresa será acrecentar al máximo el valor presente de los ingresos netos, o sea

$$
\operatorname{MaxV}=R_{2}\left(1+i_{1}\right)^{-1}-I_{1}
$$

Sustituyendo la expresión (5) en la función objetivo (6), V queda sólo como función de I, de manera que

$$
\frac{d V}{d I}=f^{\prime}\left(I_{1}\right)\left(1+i_{1}\right)^{-1}-1=0
$$

implica $f^{\prime}\left(I_{1}\right)-1=i_{1}$ ó $\rho=i_{1}$.

Por lo tanto, la empresa iguala su tasa interna de rendimiento marginal con la tasa de rendimiento del mercado correspondiente. Hasta aquí, todavía estamos en un mundo de mercados 
perfectos; ahora bien, si el mercado de capital es imperfectamente competitivo en el sentido de que una mayor inversión de la empresa i tiene un efecto positivo sobre la tasa de interés, entonces:

$$
\frac{d V}{d I_{1}}=f^{\prime}\left(I_{1}\right)\left(1+i\left(I_{1}\right)\right)-f(I)\left(1+i\left(I_{1}\right)\right)^{-2} \frac{\partial i_{1}}{\partial I_{1}}=1
$$

Dado que $i_{1}^{\prime}\left(I_{1}\right)>0$, el segundo término es positivo. En consecuencia la empresa se encontrará con que el monto de la inversión que eleva al máximo el valor de la empresa es menor que en el caso de los mercados perfectos. Esto se ilustra en la Gráfica 3.

\section{Gráfica 3}

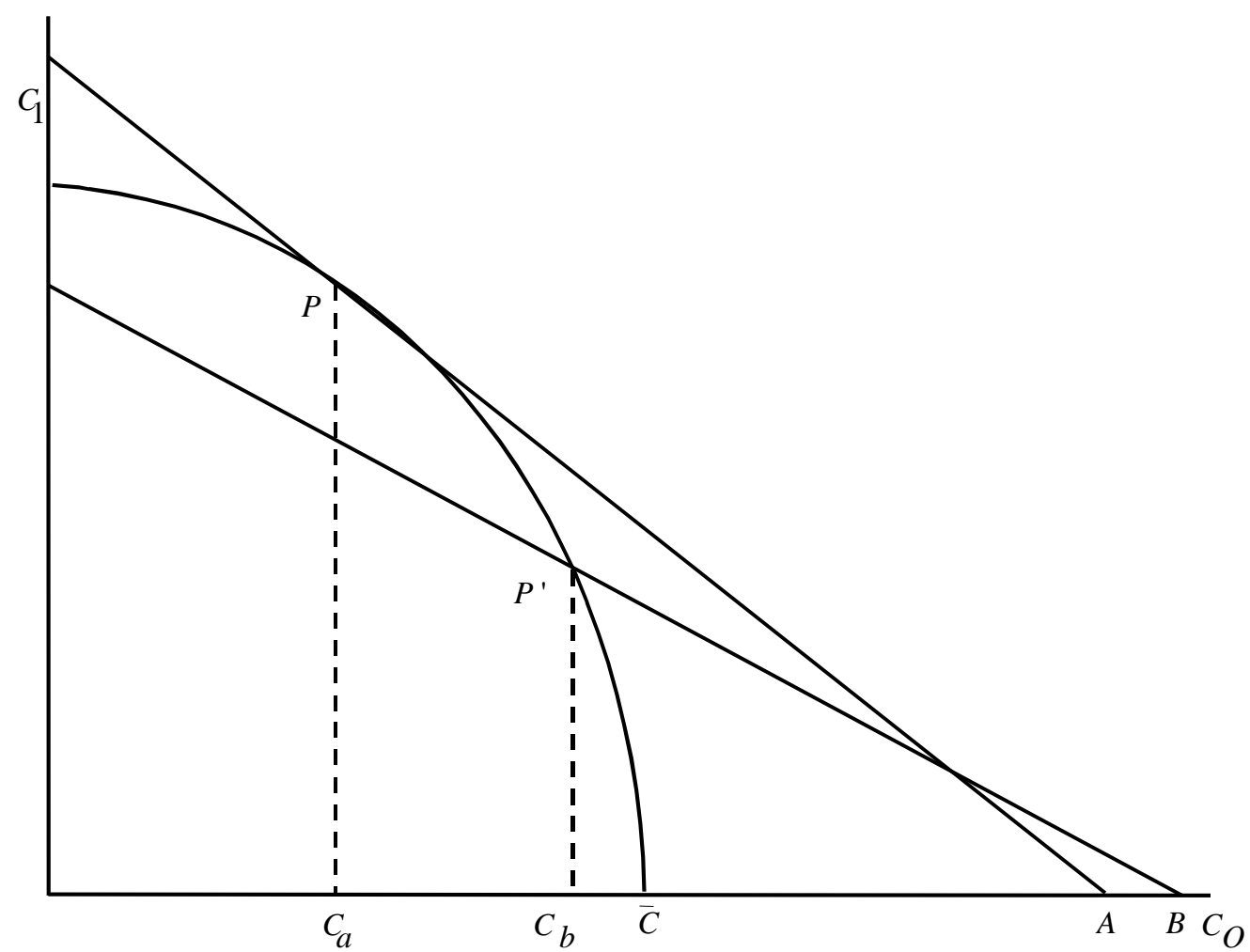

En este caso, el momento de inversión que maximiza el valor de la empresa $\bar{C}-C_{b}$ es inferior al que se realiza cuando la línea de intercambio del mercado de capital es tangente al conjunto de oportunidades de la producción, $\bar{C}-C_{a}$.

Ya no se aplica el principio básico de separación. Las decisiones de inversión dependen de las funciones de utilidad individuales, y no existe una decisión única de producción que pudieran tomar los accionistas, sin tomar en cuenta sus preferencias. Es fácil visualizar en la Gráfica 3 cómo se podrían 
ilustrar las curvas de indiferencia de dos individuos tales que uno prefiera producir un $\mathrm{P}$, mientras el segundo escogería $\mathrm{P}^{\prime}$.

\subsubsection{Mercados Imperfectos: Tasas Divergentes y Racionamiento de Capital}

Examinemos ahora un segundo caso de mercados imperfectos: cuando las tasas que se pagan a los ahorradores difieren de los que se cobran a los prestamistas. Esta situación es bastante sencilla de conceptualizar, ya que puede interpretarse como un caso de mercados perfectos en donde existe un costo fijo de transacción por unidad. La Gráfica 4 ilustra el caso en que la tasa de adquisición de préstamos es mayor que la tasa de concesión de préstamos. En este caso, las oportunidades de financiamiento amplían el conjunto de consumo factible en la magnitud representada por las áreas sombreadas.

\section{Gráfica 4}

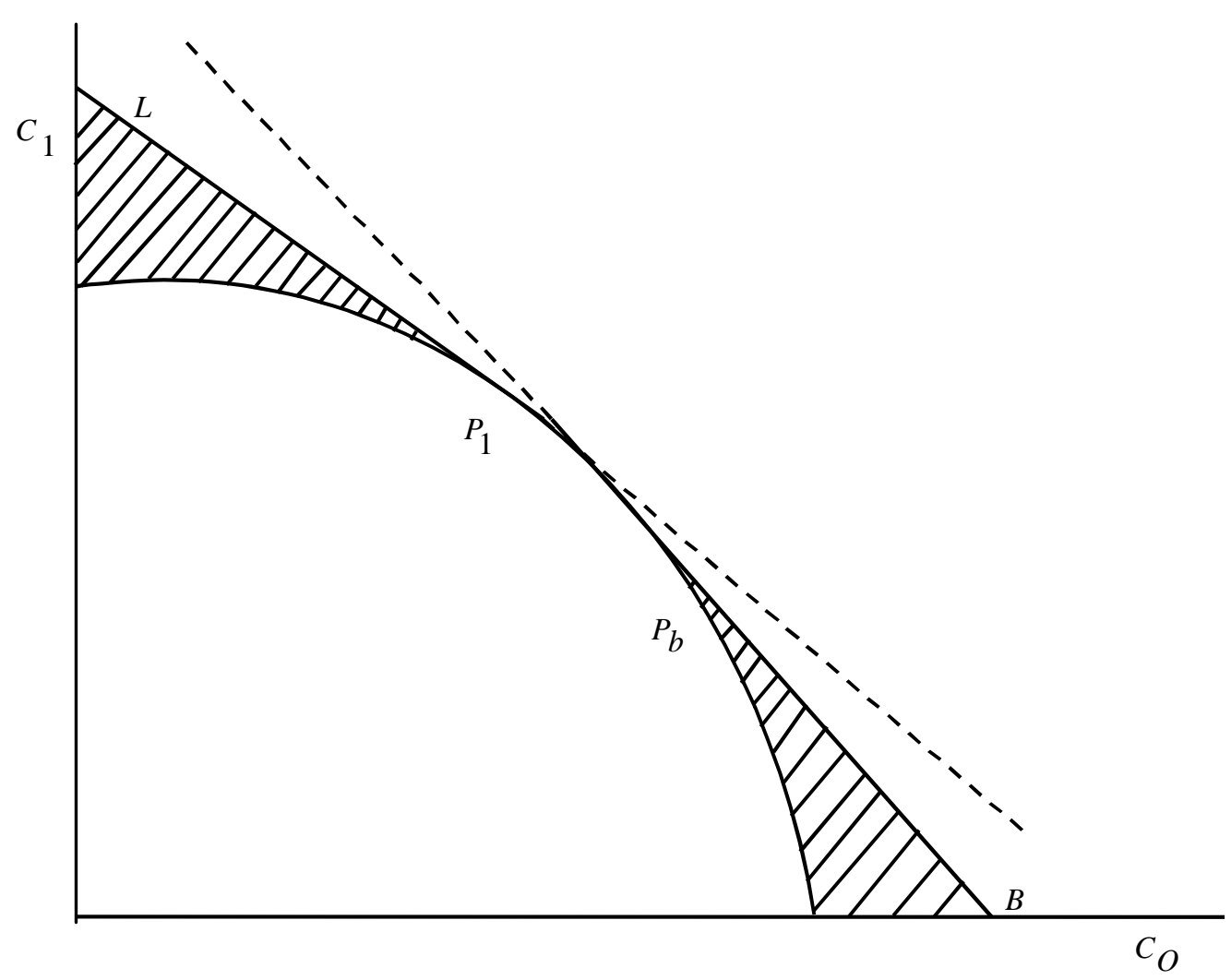

Al igual que en el ejemplo anterior, no existe una sola decisión de producción por parte de las empresas que pudiera ser aprobada unánimemente por sus propietarios, sin importar sus preferencias. Los inversionistas cuyas curvas de indiferencia son tangentes a la línea de concesión de créditos del 
mercado de capital, producirán en el punto $P_{1}$. Aquéllos cuyas preferencias sean más inclinadas al consumo actual, escogerán $P_{b}$. A la única conclusión a la que se puede llegar en este caso es que el punto de producción se encontrará entre $P_{1}$ y $P_{b}$.

Una situación más interesante para el caso de las economías en desarrollo es aquél en donde existen limitaciones cuantitativas a la disponibilidad de capital; esto significa que el capital se encuentra racionado. Basan Kapur (10) realizó un análisis detallado de este problema y demostró, bajo condiciones generales, que cuando se raciona el capital disponible de la empresa, los resultados microeconómicos tradicionales con respecto a la selección de insumos por parte de las empresas se tornan ambiguos. En particular, si existen restricciones cuantitativas a la adquisición de capital, la relación capital-mano de obra aumentará en lugar de descender.

El problema del racionamiento de capital puede incorporarse al problema general de optimización de la empresa de la siguiente manera:

$$
\begin{gathered}
\operatorname{Max} \quad p F(K, L)-\bar{r} K-w L \\
\text { s.a. } K \leq \bar{K} \quad \text { y } \quad K>0, L>0
\end{gathered}
$$

y se puede resolver empleando técnicas estándar de programación no lineal.

La condición de Khun-Tucker que se aplica para ilustrar los resultados arriba mencionados es:

$$
P \frac{F}{K}-r-\lambda=0 \quad \text { si } \quad K>0 \quad \text { y } \quad K=K^{*}, \quad L=L^{*}
$$

que difiere del caso sin restricciones por $\lambda, \mathrm{y}$ donde $r+\lambda / p$ constituye el precios "sombra" del capital. El efecto de un aumento en la tasa de interés r, cuando la restricción opera $(\lambda / p>0)$, será la reducción de la prima "sombra" del capital $(\lambda / p)$ dejando invariable el precios sombra y, por lo tanto, no afecta a la relación capital-mano de obra. Si, además de las restricciones cuantitativas, la empresa se enfrenta a costos crecientes de adquisición de préstamos, es fácil demostrar que los resultados de Kapur se mantienen. La nueva condición de primer orden es la siguiente:

$$
P \frac{F}{K}-r-\lambda-\frac{r}{K} K=0 \quad \text { si } \quad K>0 \quad \text { y } \quad K=K^{*}, \quad L=L^{*}
$$


La nueva $\mathrm{K}^{*}$ diferirá (será inferior) de la precedente, pero se aplica esencialmente el mismo razonamiento.

\subsection{Decisiones Bajo Incertidumbre.}

\subsubsection{Riesgo e Incertidumbre: Una Discusión}

Desde hace mucho tiempo la teoría económica ha tenido que recurrir al mundo de la incertidumbre para explicar las decisiones que se toman entre alternativas de acción que no pueden ser tratadas de manera satisfactoria mediante hipótesis de certidumbre.

Los conceptos de incertidumbre y de riesgo han sufrido varias transformaciones desde el trabajo precursor de Fisher y Knight. En la estructura teórica de la "selección" moderna (choice theoretic structure), ha desaparecido la antigua distinción entre el riesgo y la incertidumbre. La descripción de los conjuntos de unidades de consumo y de producción, cuyos elementos son sucesos inciertos, se efectúa sencillamente reclasificando (o renumerando) los productos. Además de sus características físicas y su localización (que describen un producto o bien de un modo de certidumbre), debe añadirse la situación o estado preciso (state of the world) en el que ocurre. Según Debreu: "resumiendo, el concepto de un bien incierto se deriva de la noción de un bien "seguro", sustituyendo la estructura en forma de árbol de los eventos por la estructura lineal de las fechas, reemplazando siempre (la palabra) "fecha" por "evento". (3 pág. 99) ${ }^{10}$. El autor procede luego a demostrar que todos los teoremas generales de equilibrio que se aplican a una economía competitiva en un mundo de certidumbre, también se aplican al caso de la incertidumbre así definida. Por consiguiente, la incertidumbre relacionada con el conjunto de oportunidades está totalmente descrita por el conjunto d los posibles estados de la naturaleza; es decir, dado el estado de la naturaleza, se resuelve toda incertidumbre. Sería útil, sin embargo, seguir brevemente la evolución del concepto de Knight acerca de la incertidumbre hasta llegar al enfoque actual de los "estados de la naturaleza" de Arrow y Debreu.

Según Knight, el concepto de incertidumbres crucial para reconciliar la teoría económica con realidad. Knight considera que la ausencia de competencia en los mercados, y la aparición de ganancias excesivas, está relacionada con los cambios en las condiciones económicas imperantes producidas éstas por la existencia de incertidumbre. Las utilidades provienen del hecho que los

\footnotetext{
${ }^{10}$ Un evento es un conjunto de "estado o situaciones" de la naturaleza (states of the world). Se definirá más adelante. Ver Arrow (1), pág. 46. El texto original es "Summing up, the concept of uncertain commodity is derived from the concept of
} 
empresarios contratan la compra de servicios productivos antes de vender el producto final. Así, la competencia por servicios productivos se basa en anticipaciones, mientras que la venta del producto final está sujeta a eventos inciertos. Considera que los sucesos inciertos generalmente se pueden catalogar en tres categorías principales ${ }^{11}$ :

1. Probabilidades a priori: aquéllas para las cuales se puede calcular con principios generales la verosimilitud de un suceso. Por ejemplo: obtener el número seis al arrojar un dado.

2. Probabilidad estadística: sólo se puede calcular en forma empírica. Aun cuando es viable un alto grado de confiabilidad en las estimaciones que se obtienen a través de este método, la verdad es que los juicios están fundamentados sobre una base a priori indeterminada.

3. Estimaciones: juicios para los que existe una base de clasificación válida. Se hacen estimaciones de sucesos inciertos cuyos resultados no existe dentro de la esfera de acción de los juicios probabilísticos. Las situaciones en las que se aplican estas estimaciones son singulares en cierto modo.

Basándose en esta clasificación, Knight hace la distinción entre el riesgo y la incertidumbre. El riesgo se utiliza para describir situaciones en las que se conoce la distribución del resultado ya sea a priori o estadísticamente. La incertidumbre se refiere a una categoría más extensa (estimaciones), en la que es imposible hacer una clasificación con fines estadísticos debido a la singularidad de la situación.

La hipótesis de que las estimaciones difieren de las probabilidades estadísticas en el hecho de que no existen principios válidos de clasificación para las primeras, conduce, según Arrow (1), hacia la formulación moderna del problema estadístico efectuada por Neyman y Pearson.

Examinemos cualquier suceso incierto. Existe un conjunto conocido de hipótesis viables, una de las cuales se sabe que es verdadera. No hay juicios probabilísticos que nos permitan diferenciarlas, de modo que se eliminan las probabilidades a priori. Es posible realizar experimentos (por la naturaleza o por el estadístico) cuyo resultado sea una variable aleatoria con una distribución determinada de probabilidades. Con base en el resultado, se adoptan medidas cuyas consecuencias dependen de las mismas medidas tomadas y de la hipótesis verdadera. Entonces, a cada medida

certain commodity by substituting the true structure of events for the line structure of dates and replacing everywhere (the word) "date by event".

${ }^{11}$ Ver Knight (11) Cap. 7. 
adoptada podemos asociar una función de "ingresos" o de "pérdida" que especifique para cada hipótesis verdadera posible cuáles serán las consecuencias de la acción.

Bajo esta formulación, el problema de selección consiste en escoger entre las distintas funciones de ingreso. Esta es la estructura teórica básica que sirve de fundamento al enfoque de los "estados de la naturaleza". Cabe señalar que únicamente hemos hablado de un aspecto de la teoría de la selección en condiciones inciertas: a la descripción del problema y a la caracterización del concepto de incertidumbre. No se ha dicho nada acerca de la teoría de la conducta; esto es, la forma en la que se ordenan y evalúan en una función de preferencias las consecuencias de las acciones emprendidas. No obstante, este es un aspecto de la teoría que no nos concierne por el momento.

La construcción de una teoría especial de selección bajo condiciones inciertas, empleando el lenguaje estadístico presentado anteriormente, es necesaria por el hecho de que una acción no debe producir, necesariamente, una consecuencia única. Por esto, un ordenamiento de las consecuencias no determina selecciones únicas entre las acciones como sucede con el caso de la certidumbre, donde la relación es de uno a un ${ }^{12}$. El concepto de los estados de la naturaleza es entonces una descripción completa del espacio, d manera que se logran determinar las consecuencias de cada acción. La incertidumbre ligada con el conjunto de oportunidades de los agentes económicos está conformada completamente por el conjunto de los posibles estados de la naturaleza; es decir, dado un estado de la naturaleza, se resuelve cualquier incertidumbre. Una característica importante de este modelo es el hecho de que el individuo no puede influir en el resultado de las acciones. La naturaleza es la que determina este resultado.

No sólo tienen los individuos un completo conocimiento de los resultados condicionales (o funciones de ingreso), sino que también dentro del marco de equilibrio general, todos deben de estar de acuerdo con la división de la naturaleza (o del mundo) en estados específicos. ¿Qué tan detallada debe ser esta división?. Debe de ser tan minuciosa que permita que las preferencias de todos los participantes, las funciones de producción de todas las empresas, y los rendimiento de cada uno de los valores, estén definidos singularmente en cada estado. Como lo señaló Hirshleifer (9), estos supuestos implican que el modelo se refiere únicamente a lo que él llama la "incertidumbre productiva"...”Se deben de diferenciar claramente dos tipos de incertidumbre: incertidumbre productiva e incertidumbre en las transacciones. La incertidumbre productiva se refiere al desconocimiento del resultado de sucesos naturales exógenos que podrían ser interesantes hasta para un Robinson Crusoe que se 
encuentra aislado de las oportunidades de mercado... La incertidumbre en las transacciones, por otra parte, se refiere a las oportunidades de intercambio. Representa el desconocimiento del individuo de que la curva efectiva de oferta-demanda de otros sujetos. Con este último tipo de incertidumbre, los comerciantes potenciales incurrirán típicamente en costos de operación al buscar con quién intercambiar; el resultado de esta búsqueda es una variable aleatoria. Por consiguiente, desde el punto de vista sujeto, el mercado es imperfecto, en virtud de que desconoce de antemano un precio único al que se podrían efectuar todas las transacciones" (9 pág. 243).

Entonces, es aparente que la forma en la que se describe a la incertidumbre en el modelo de Arrow_Debreu excluye la posibilidad de que existan mercados imperfectos.

Es evidente que en el mundo real no existen los "certificados de Arrow" en forma pura ${ }^{13}$. Lo que observamos que se comercia en el mercado de capital son acciones y valores que normalmente no ofrecen rendimientos que dependan de la ocurrencia de estados de la naturaleza particulares. Si el número de valores existente en el mercado es inferior al monto total de los posibles estados de la naturaleza, el mercado de capital estará incompleto. Por otra parte, en la literatura financiera se ha determinado que comerciar con un número limitado de valores (mercados incompletos) dará como resultado distribuciones subóptimas de riesgos. La regla de valuación del "óptimo de Pareto" tendrá que remplazarse por la del "óptimo restringido de Pareto" (Constrained Pareto Optimality) ${ }^{14}$.

El concepto de un mercado de capital incompleto se maneja en forma muy diferente al concepto de un mercado de capital imperfecto en la literatura especializada. Ya se explicó anteriormente que un mercado incompleto es aquel en el que el número de valores que existen es inferior al número de estados de la naturaleza; sin embargo, se supone que cuando existen mercados, estos se comportan en forma competitiva. Imaginemos una economía cuyas actividades se extienden sobre tres intervalos de tiempo, y en donde la incertidumbre existente proviene de la selección que efectúa la naturaleza entre un número finito de alternativas.

\footnotetext{
${ }^{12}$ Ver Arrow (1), Cap. 2

${ }^{13}$ Los certificados de Arrow son un conjunto de valores, uno para cada estado de la naturaleza, que pagarán una unidad del numerario se llega a presentar dicho estado en particular, y no se pagarán en el caso de que no ocurra el estado. Estos valores fueron sugeridos inicialmente por Arrow (2), y por consiguiente, se denominan certificados Arrow

${ }^{14}$ Una distribución será "Pareto óptima restringida" si la autoridad central, operando en los mercados disponibles, no puede mejorar la situación de cualquier persona sin empeorar la de otra (Stiglitz (23). Este concepto fue introducido originalmente por P. Diamond (4)).
} 
Gráfica 5

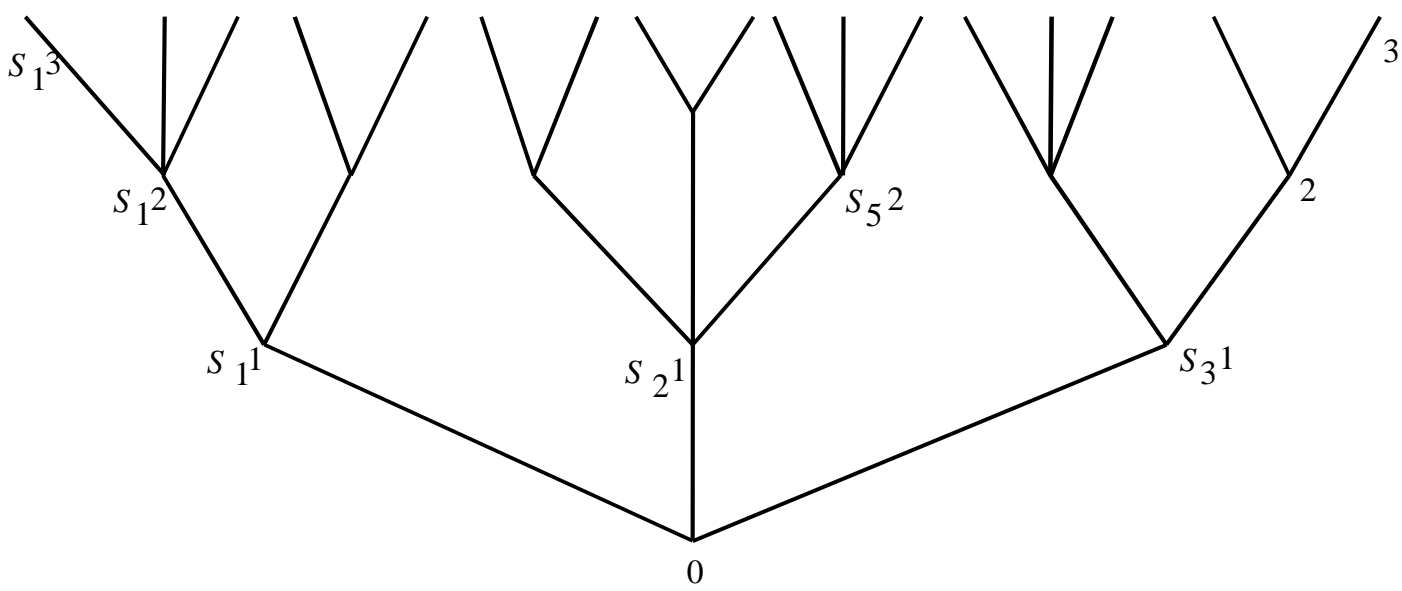

Cada uno de los vértices del árbol de eventos $S_{t}^{t}$ de la Gráfica 5 (donde el subíndice se refiere al estado de la naturaleza en el periodo $\mathrm{t}=1,2,3)$ representa un determinado pago en dinero. Supongamos también que existen mercados perfectos para todos lo valores que se van a vender (uno para cada estado de la naturaleza en $\mathrm{t}=1,2,3$ ) exceptuando un mercado, digamos $S_{5}^{2}$. En este mercado particular, los problemas de información o los costos de operación evitan que prevalezca un precios único. ¿Podemos concebir que en este modelo surja una situación de este tipo?. La respuesta es no, ya que estaríamos introduciendo "incertidumbre por el lado de las transacciones". Aquí ya no es la naturaleza quien escoge. Entonces, lo apropiado en esta caso es dividir aun más los estados de la naturaleza en $\mathrm{t}=2$ hasta que se elimine totalmente este tipo de incertidumbre.

El propósito del ejemplo anterior era el de ilustrar las dificultades que se presentan cuando se pretenden tratar situaciones de mercados imperfectos empleando este modelo. Sin embargo, la mayor parte d la literatura sobre finanzas e incertidumbre utiliza precisamente este modelo (estados de la naturaleza) o el de fijación de precios de activos fijos (capital asset pricing model). A pesar de que este último modelo es un caso especial del primero, se desarrolló independientemente del enfoque de los estados de la naturaleza y se ha empleado ampliamente en el análisis de la decisión de inversión que enfrenta una empresa bajo condiciones de incertidumbre. Originalmente fue Sharpe (18) quien propuso este modelo, y fue generalizado por Linter y Mossin (12). 


\subsubsection{Decisiones de la Empresa}

Se mencionó anteriormente que la regla de conducta para la empresa que se desprende del caso Fisheriano de certidumbre es la de maximizar el valor presente de la empresa (o sea de las acciones emitidas). Esta regla es el resultado del teorema básico que antes discutimos. Cuando existe incertidumbre, sin embargo, la regla de maximizar el valor presente de la empresa topa con algunas dificultades. Una de ellas es determinar en que forma se debe de calcular el valor presente ${ }^{15}$. Por otra parte, bajo condiciones de incertidumbre, el principal problema no es tanto el especificar la regla de conducta que norma las decisiones de la empresa, sino la posibilidad de aplicar el criterio tradicional de valorización de bienestar de Pareto.

El problema estriba en saber si las decisiones que toman las empresas satisfacen el criterio de Pareto. Si la maximización del valor presente es la regla de conducta aceptada, se puede formular el problema de manera más concreta, averiguando si las empresas que maximizan el valor presente de sus activos netos toman decisiones óptimas en le sentido de Pareto. Existen dos casos en los que se han demostrado rigurosamente que las empresas toman decisiones en el sentido mencionado:

- En condiciones de certidumbre y de mercados perfectos (caso Fisheriano).

- Bajo incertidumbre, si las empresas son "aceptadoras" de precios en un mercado de capital perfecto y completo. Esto sucede cuando la incertidumbre sólo se relaciona con la ocurrencia de "estados de la naturaleza" bien definidos, para los que existe un conjunto completo de certificados de Arrow-Debreu16.

Anteriormente hicimos referencia al hecho de que no existen los certificados Arrow_Debreu y que probablemente encontramos mercados de capital incompletos en el mundo real. Lo que observamos son valores ordinarios y acciones que no ofrecen distintos rendimientos para cada uno de los distintos estados de la naturaleza. Con el modelo de "media-varianza" pueden examinarse situaciones de mercado incompletos, y frece una regla simple para tratar variables aleatorias: transforma magnitudes inciertas (ingresos, rendimientos, etc.,.) en equivalentes de certidumbre. Esto

\footnotetext{
${ }^{15}$ Los dos enfoques básicos son: construir una tasa de descuento "con riesgo" mediante el aumento de primas de riesgo a la tasa de interés, y el crear equivalentes de certidumbre para entradas inciertas de efectivo en el futuro. El segundo enfoque se utiliza en el modelo de la fijación de precios de los activos fijos (capital asset pricing model). Ver Sharpe (19). Todavía se discute si en la ausencia de los certificados de Arrow-Debreu, el objetivo correcto de la empresa es el de maximizar el valor presente. Ver Stiglitz (22).
} 
explica por qué se utiliza tanto este modelo. Si se introducen más hipótesis, como la existencia de activos que no presentan riesgos y de expectativas homogéneas con respecto al valor futuro de los valores que se comercian, se obtiene el modelo de fijación de precios sobre activos fijos (capital asset pricing model). En el contexto de este modelo, Stiglitz (23), Fama (5) y otros autores arguyen que la distribución del riesgo, que es el resultado de las actividades de empresas que maximizan el valor presente, no será la óptima en le sentido de Pareto. Aun cuando se han planteado algunas dudas sobre la generalidad de estos resultados, ahora se acepta que únicamente con hipótesis muy restrictivas sobre las preferencias de los inversionistas puede un mercado competitivo de acciones y abonos dar como resultado una distribución óptima en el sentido de Pareto ${ }^{17}$.

\subsubsection{Mercados Imperfectos: Restricciones Cuantitativas}

Para simplificar, imaginemos una economía en la que el valor de mercado de todas las empresas existentes no está correlacionado. Entonces, de acuerdo a Stiglitz (23), el valor de la empresa j es:

$$
V_{j}=\frac{E X_{j}-\psi \sigma_{j}^{2}}{r}
$$

donde $E X_{j}$ y $\sigma_{j}^{2}$ son, respectivamente, la media y la varianza de la producción de la empresa $j ; r$ es la tasa de rendimiento sin riesgo (es igual a uno más la tasa de interés del activo que no representa riesgo) y $\psi$ es el factor de corrección de riesgo ${ }^{18}$. Si la empresa maximiza el valor presente, $V_{j}-I_{j}$, ( $I_{j}$ es la inversión que efectúa la empresa $j$ ), las condiciones de primer orden son:

$$
\begin{aligned}
& \frac{\partial E X_{j}}{\partial I_{j}}-2 \psi \sigma_{j} \frac{\partial \sigma_{j}}{\partial I_{j}}=r \\
& \frac{\partial E X_{j}}{\partial I_{i}}=r
\end{aligned}
$$

\footnotetext{
${ }^{16}$ Ver Stiglitz (23) y Mossin (15) Cap. 6

${ }^{17}$ En una tesis muy cuidadosa, N. Nielsen analiza detalladamente el trabajo de Stiglitz, Fama, Jensen y Long y de otros autores. Puede demostrar casos particulares de mercados de capital incompletos, en el contexto del modelo de fijación de precios de activos fijos, donde las decisiones de las empresas que maximizan el valor presente de sus activos, resultan en distribuciones óptimas en el sentido de Pareto.

18 ver, por ejemplo, el texto de Mossin (14), o la tesis de Nielsen (16).
} 
donde la empresa $i$, representa la empresa que no incurre en ningún riesgo (riskless firm). Si estas condiciones se derivan directamente de las funciones de utilidad del consumidor (suponiendo gustos idénticos), las condiciones de primer orden son:

$$
\begin{aligned}
& \frac{\partial E X_{j}}{\partial I_{j}}-\psi \sigma_{j} \frac{\partial \sigma_{j}}{\partial I_{j}}=r \\
& \frac{\partial E X_{i}}{\partial I_{i}}=r
\end{aligned}
$$

Al comparar (12) y (13) encontramos que las empresas que maximizan el valor presente valúan el riesgo al doble de lo que indicaría una distribución óptima de Pareto. Stiglitz subraya el resultado será una subinversión (desde el punto de vista del mercado) en las empresas que conllevan riesgo en relación con las empresas sin riesgo.

Si la empresa se enfrenta a restricciones cuantitativas sobre el monto que puede tomar prestado con fines de inversión, el problema se puede expresar de la siguiente manera:

$$
\operatorname{Max}\left(V_{j}-I_{j}\right)
$$

$$
\text { s.a. } \quad I_{i \leq} \bar{I} \quad, \quad I_{j} \geq 0 .
$$

Las condiciones de primer orden (Khun-Tucker) son:

(15) si $I_{j}^{*}=0, \quad$ entonces $\frac{\partial E X_{j}}{\partial I_{j}}-2 \psi_{j} \frac{\partial \sigma_{j}}{\partial I_{j}}-\lambda=r \quad$ en el punto $I_{j}=I_{j}^{*}$

(16) si $\quad I^{*}=0, \quad$ entonces $\frac{\partial E X X_{j}}{\partial I_{j}}-2 \psi \sigma_{j} \frac{\partial \sigma_{j}}{\partial I_{j}}-\lambda \leq r$ en el punto $I_{j}=I^{*}$

(17) si $\lambda>0$, entonces $\quad I_{j}-\bar{I}=0$

(18) si $\lambda=0, \quad$ entonces $\quad I_{j}^{*}-\bar{I} \leq 0$

$$
I^{*} \geq 0 \quad \lambda^{*} \geq 0
$$


Si se efectuará la inversión y las restricciones de capital son efectivamente limitantes, las condiciones relevantes son la (15) y la (17). Es interesante observar que en el caso de las empresas cuyos rendimientos no están correlacionados, la valoración de riesgos que realizan el mercado no se altera si al empresa enfrenta restricciones cuantitativas sobre el monto de financiamiento que puede adquirir. El peso de la restricción de capital recae sólo en la empresa que no conlleva riesgo (riskless firm).

Esto implica que, dado el volumen total de la inversión en el mercado, la imposición de una restricción de capital reducirá la proporción de los fondos asignados a la empresa sin riesgo. En al gráfica 6, el conjunto de oportunidades en el espacio $\left(E x, \sigma_{x}\right)$ representa el conjunto de carteras eficaces $^{19}$. La condición de equilibrio del mercado que obtuvo Stiglitz es que la pendiente del "locus" de las oportunidades en cualquier punto es el doble de la pendiente de la curva de indiferencia corresponderá a dicho punto en la frontera de posibilidades. El punto A corresponde a la situación óptima ideal de Pareto; el punto B, al equilibrio que obtuvo Stiglitz, y en el punto C, al que obtuvo en el caso restringido. Si se supone que las empresas reciben rendimiento que no son independientes, los resultados no cambian, en tanto que las medidas adoptadas por la empresa no afecten la tasa de interés (sin riesgo) y el factor de corrección del riesgo.

\footnotetext{
${ }^{19}$ Una cartera $\mathrm{P}$ es eficiente, si ninguna cartera con el mismo rendimiento esperado, $\mathrm{E}(\mathrm{P})$, o uno mayor, tiene una desviación estándar inferior.
} 
Gráfica 6

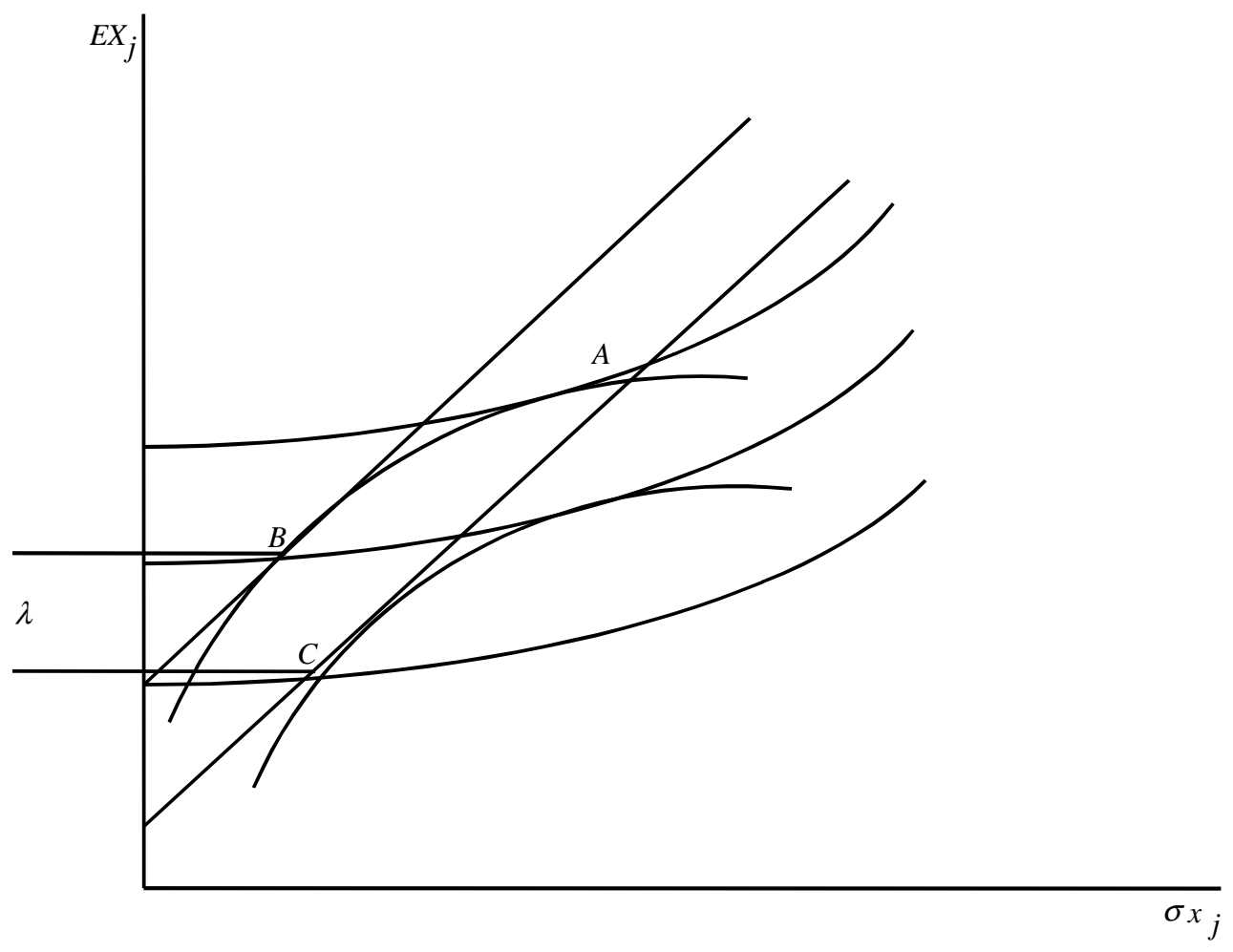

\subsubsection{Mercados Imperfectos: Costos Crecientes de la Adquisición de Préstamos}

Antes de examinar este caso, es necesario subrayar que si los mercados de capital no son perfectos, no hay razón para creer que el objetivo apropiado para una empresa sea maximizar su valor presente. En el caso en que la empresa enfrenta restricciones cuantitativas, descrito anteriormente, la incapacidad de la empresa $r$ y $\psi$, indica que ésta se comporta como una aceptadora de precios. Si embargo, si la decisión de inversión de una empresa afecta a la tasa de interés (de modo que el costo de capital aumenta con el nivel de la inversión), entonces dicha empresa no está operando ya en un mercado competitivo, y la situación se torna más complicada. Una vez más, en el contexto del modelo de la fijación de precios de los activos fijos, el valor de la empresa $j$ es:

$$
V_{j}=\frac{E X_{j} \psi \sum_{i+j} \sigma_{i j}}{r\left(I_{j}\right)}=\frac{E X_{j}-\psi\left(\sigma_{j}^{2}+\sum_{i+j} \sigma_{i j}\right)}{r\left(I_{j}\right)}
$$

donde ${ }_{i j}$ es la suma de las covariaciones de las empresas $n-1$ con la empresa $j$. Si la empresa maximiza el valor presente neto, la condición de primer orden es: 
$\frac{1}{r\left(I_{j}\right)^{2}}\left[r\left(I_{j}\right)\left(\frac{\partial E X_{j}}{\partial I_{j}}-\psi\left(2 \sigma_{j}+\sum_{i+j} \frac{\partial \sigma_{i j}}{\partial I_{j}}\right)-\left(\sigma_{j}^{2}+\sum_{i+j} \sigma_{i j}\right) r^{\prime} \frac{\partial \psi}{\partial I_{j}}\right)-\left(E X_{j}-\psi\left(\sigma_{j}^{2}+\sum_{i+j} \sigma_{i j}\right)\right) r^{\prime}\left(I I_{j}\right)\right]=1$

El último término $E X_{j}-\psi\left(\sigma_{j}^{2}+\sum_{i+j} \sigma_{i j}\right) r^{\prime}\left(I_{j}\right) / r\left(I_{j}\right)^{2}$ es simplemente $r^{\prime}\left(I_{j}\right) / r\left(I_{j}\right) V_{j}$, de manera que la expresión (21) se puede expresar:

$$
\frac{\partial E X}{\partial I_{j}}-\psi\left(2 \sigma_{j}+\sum_{i+j} \frac{\partial \sigma_{i j}}{\partial I_{j}}\right)-\left(\sigma_{j}^{2}+\sum_{i+j} \sigma_{i j}\right) r^{\prime}\left(I_{j}\right) \frac{\partial \psi}{\partial I_{j}}-V_{j} r^{\prime}\left(I_{j}\right)=r
$$

que difiere del caso del mercado perfecto debido a la presencia del tercero y cuarto términos en $(22)^{20}$. El último término es evidentemente positivo en tanto $r^{\prime}\left(I_{j}\right)>0$. El tercer término debe examinarse más de cerca. El factor de correlación de riesgo puede expresarse:

$$
\psi=\frac{E M-r \sum_{j} V_{j}}{\sigma_{M}^{2}}=\frac{E \sum_{j} X_{j}-r_{j} V_{j}}{\sigma_{M}^{2}}
$$

donde $M=\sum_{j} X_{j}$, de manera que

$$
\frac{\partial \psi}{\partial I_{j}}=\left[E \sum_{j} \frac{\partial X_{j}}{\partial I_{j}}-r \sum_{j} \frac{\partial V_{j}}{\partial I_{j}}-\sum_{j} V_{j} r^{\prime}\left(I_{j}\right)\right] \frac{1}{\left(\sigma_{M}^{2}\right)^{2}}-\left(E \sum_{j} X_{j}-r \sum_{j} V_{j}\right) \frac{\partial \sigma_{M}^{2}}{\partial I_{j}} \frac{1}{\left(\sigma_{M}^{2}\right)^{2}}
$$

El signo de $\partial \psi / \partial I_{j}$ será positivo si la primera expresión entre paréntesis es positiva y mayor que la segunda si la segunda expresión es negativa). Simplemente observando la expresión (23) resulta claro que, a menos se formulen hipótesis específicas para restringir a priori los signos de los diferentes términos, no se puede llegar a una conclusión en relación con el efecto total sobre el factor de

\footnotetext{
${ }^{20} \mathrm{Si}$ se suponen mercados perfectos, la condición de primer orden que resulta de maximizar al valor presente neto para el caso en que los rendimientos de las distintas empresas no son independientes es:

$$
\frac{\partial E X_{j}}{\partial I_{j}}-\psi\left(2 \sigma_{j}+\frac{\partial \sigma_{j}}{\partial I_{j}}+\sum_{i+j} \frac{\partial \sigma_{i j}}{\partial I_{j}}\right)
$$
}


corrección de riesgo del mercado producido por un cambio en la decisión de inversión de la empresa. Es decir, se tienen que hacer hipótesis específicas sobre el comportamiento de las empresas (y sus funciones de reacción).

Por ejemplo, el primer término $\partial E M / \partial I{ }_{j}$ representa el efecto que tiene un incremento de la inversión de la empresa $j$ sobre la oferta total, Si $\partial E M / \partial_{j}>0$, el signo del término $\partial X_{j} / I_{j}$ tiene que ser negativo para alguna $i$, si la decisión de la empresa $j$ de producir necesita algún insumo. El segundo término $\sum \partial V_{j} / \partial I$ indica que el efecto que tiene sobre la valoración del mercado de las $n$ empresas, un cambio en la decisión de invertir de la empresa $j$. Otra vez, se necesitan más restricciones para determinar el signo de esta expansión.

Sin embargo, existe una segunda interpretación para el factor de corrección de riesgos, que puede ofrecernos una mejor perspectiva del problema. $\psi$ también se puede interpretar, en el contexto del modelo de la fijación de precios de los activos fijos, como el precios del riesgo en el mercado en el siguiente sentido ${ }^{21}$ : pensemos en pares de valores $E X_{j}$ y $\sigma_{i j}$ para los que $V_{j}$ es igual. Diferenciando totalmente la ecuación (20) y estableciendo que $d V_{j}=0$,

$$
\psi=\frac{d E X_{j}}{d \sigma_{i j}} .
$$

$\psi$ mide el incremento en el rendimiento esperado que se requiere para compensar el incremento de una unidad en la de riesgo percibido por la empresa $\left(\sigma_{i j}\right)$. Si, por ejemplo, se suponen funciones de utilidad cuadráticas, es razonable esperar que el signo de $\partial \psi / \partial I_{j}$ sea positivo, debido a la aversión hacia el riesgo que implica este tipo de función de utilidad.

En este caso particular, el tercer término de la ecuación (22) será negativo. Esto implica que las empresas exigirán rendimientos más altos por cada unidad de riesgo que se tome bajo estas hipótesis. Es decir, las empresas atribuirán un costo más alto al riesgo que corren, que dará como resultado una subinversión en las industrias "riesgosas". De la ecuación (13) se desprende que para una decisión de inversión óptima, la relación entre riesgo y rendimiento esperado es lineal; sin embargo, cuando se

\footnotetext{
${ }^{21}$ Ver Mossin (15), Cap. 4.
} 
asume una conducta de aceptación de precios, se pierde el carácter lineal como se representa en la gráfica 7.

\section{Gráfica 7}

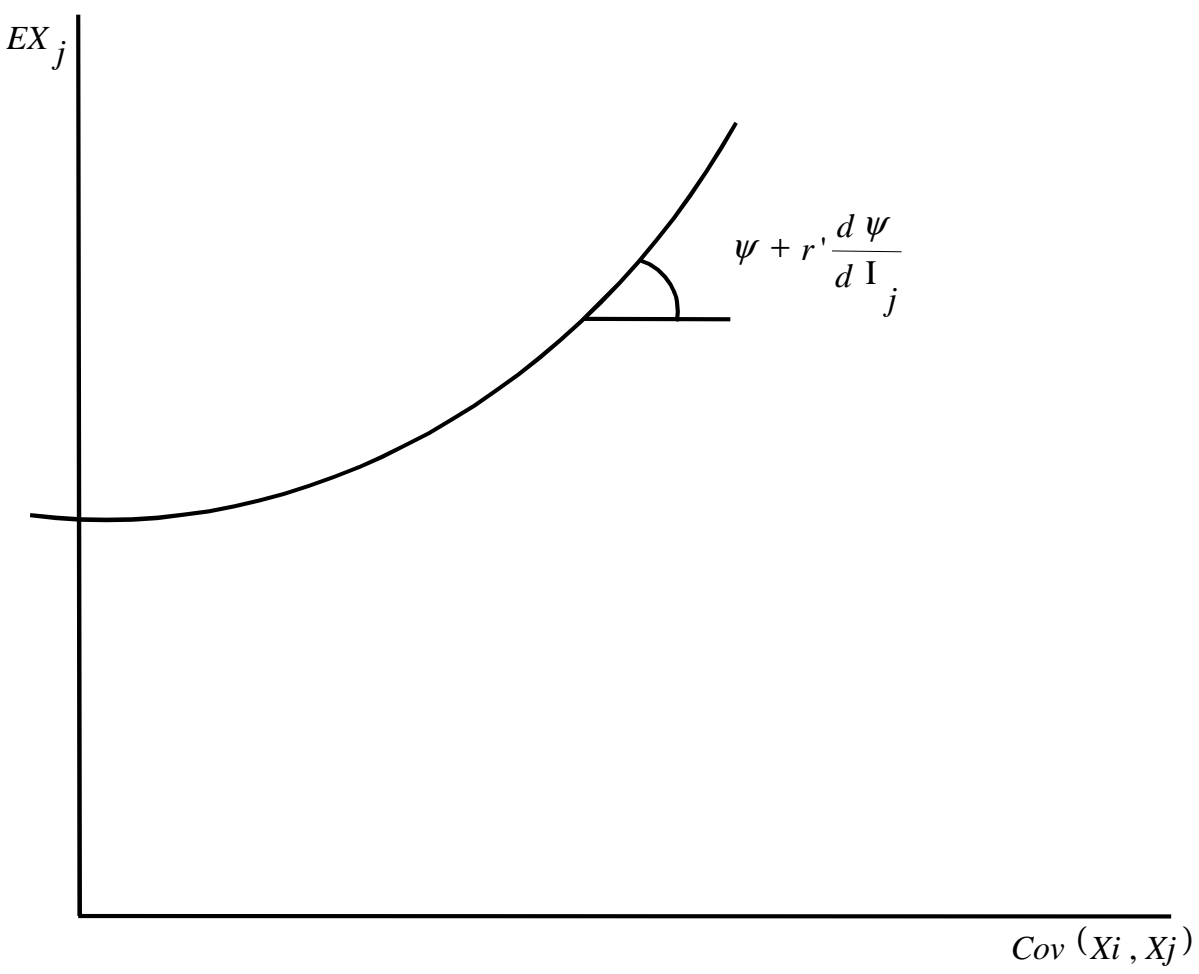

\subsubsection{Decisiones de los Consumidores}

El caso de los mercados imperfectos para la empresa que se analizó en la última sección ilustra claramente un punto que se tocó anteriormente: la dificultad para obtener resultados generales cuando se elimina la hipótesis de la existencia de un mercado de capitales perfecto. El propósito de esta sección es examinar las implicaciones que representa la existencia de mercados imperfectos de capital para el problema del consumidor.

Consideremos un modelo de dos períodos en donde el objetivo del individuo es maximizar la utilidad esperada, definida como el consumo en los períodos uno y dos.

$$
\operatorname{Max} E\left[U_{K}\left(c_{1}, c_{2}\right)\right]
$$

El consumo del período dos es una variable aleatoria cuyo valor depende del ingreso del individuo $k$ en el período dos. Si el individuo tiene una dotación inicial de $c_{1}$ unidades de consumo y 
de acciones de las $n$ empresas, ( $\bar{\beta}_{k j}$ es la proporción de la empresa $j$ que pertenece al sujeto $k$ como la parte de su dotación), y $X_{j}(\theta)$ es la producción de la empresa $j$ que depende del estado de la naturaleza $\theta$, el problema se reduce a seleccionar las $\beta_{j}$ que maximizan el valor del ingreso en el segundo período sujeto a la restricción presupuestal correspondiente:

$$
L=\left[U_{k}\left(c_{1}, \sum_{j} \beta_{k} X_{j}(\theta)\right)\right]-\lambda\left[\sum_{j} V_{j}\left(\bar{\beta}_{k j}-\beta_{k j}\right)+\bar{c}_{1}-c_{1}\right]
$$

Se puede observar el impacto que produce un cambio en la inversión de la empresa $i$ sobre la utilidad del consumidor $k$ diferenciando $L$ con respecto a $I_{i}$.

$$
\begin{aligned}
\frac{\partial I}{\partial I_{i}}=\sum_{\theta} & {\left[U_{k 1} \frac{\partial c_{1}}{\partial I_{i}} U_{k 2} \sum_{j}\left(\beta_{k j} \frac{\partial x_{j}(\theta)}{\partial I_{i}}+\frac{\partial \beta_{k i}}{\partial I_{i}}\right) X_{j}(\theta)\right] F_{k}(X) } \\
& -\frac{\partial \lambda_{k}}{\partial I_{i}}\left[\sum_{j} V_{j}\left(\bar{\beta}_{k j}-\beta_{k j}\right)+\bar{c}_{1}-c_{1}\right]-\lambda\left[\sum_{j} \frac{\partial V_{j}}{\partial I_{i}}\left(\bar{\beta}_{k j}-\beta_{k j}\right)-V_{j} \frac{\partial \beta_{k j}}{\partial I_{i}}-\frac{\partial c_{1}}{\partial I_{i}}\right] .
\end{aligned}
$$

Si se supone que las funciones de utilidad satisfacen el postulado de saciedad, entonces:

$$
\sum_{j} V_{j}\left(\bar{\beta}_{k j}-\beta_{k j}\right)+\bar{c}_{1}-c_{1}=0
$$

Si el consumidor optimiza sus tendencias de valores (maximizando $L$ ), las condiciones de primer orden implican:

$$
\begin{aligned}
& \sum_{\theta} U_{k 1} f(x)=-\lambda \\
& \sum_{\theta} U_{k 2} X_{j}(\theta) f(x)=-\lambda V_{j} .
\end{aligned}
$$

La condición (27) hace que desaparezca el término medio de (26) y las ecuaciones (28) facilitan las siguientes simplificaciones:

$$
\sum_{\theta} U_{k 1} \frac{\partial c_{1}}{\partial I_{i}} f(x)+\lambda \frac{\partial c_{1}}{\partial I_{i}}=0
$$




$$
\sum_{\theta} U_{k 2} \sum_{j} \frac{\partial \beta_{k j}}{\partial I_{i}} X_{j}(\theta) f(x)-\lambda \sum_{j} \frac{\partial \beta c_{k j}}{\partial I_{i}} V_{j}=0
$$

por que el equilibrio $V_{j}=\sum_{\theta} X_{j}(\theta) f(X)$.

La ecuación (26) se puede expresar entonces:

$$
\frac{\partial L}{\partial I_{i}}=\sum_{\theta} U_{k 2} \sum_{j} \beta_{k j} \frac{\partial X_{j}(\theta)}{\partial I_{i}}-\lambda \sum_{j} \frac{\partial V_{j}}{\partial I_{i}}\left(\bar{\beta}_{k j}-\beta_{k j}\right)
$$

La primera expresión del lado derecho se puede separar de la siguiente forma:

$$
\sum_{\theta} U_{k 2} \beta_{k i} \frac{\partial X_{j}(\theta)}{\partial I_{i}}+\sum U_{k 2} \sum_{i+j} \beta_{k j} \frac{\partial X_{j}(\theta)}{\partial I_{i}} .
$$

El primer término es el efecto sobre la función de utilidad del consumidor que produce un cambio en la producción de la empresa. El segundo, expresa el efecto sobre la utilidad esperada del inversionista de los cambios en la producción de todas las demás empresas, provocado porque la empresa $i$ cambió su decisión de invertir. Una vez más, debemos estructurar el problema en una forma específica con el objeto de determinar la dirección que sigue el cambio en la utilidad, que a su vez debe afectar la demanda de diferentes activos que forman la cartera.

El segundo término en (29) mide el "efecto riqueza". $\lambda$ es el precio "sombra" de la restricción presupuestal, que debe ser positivo. Esta expresión tendrá un signo positivo si:

$$
\sum_{j} \frac{\partial V_{j}}{\partial I_{i}} \beta_{k j}>\sum_{j} \frac{\partial V_{j}}{\partial I_{i}} \bar{\beta}_{k j}
$$

Es decir, si el cambio en le valor de la cartera óptima excede al cambio en el valor de la dotación, el cambio en la utilidad esperada del inversionista en virtud de un cambio en la variable de decisión de la empresa $i$, depende del efecto directo sobre la producción de la empresa $i$ y de la respuesta de las demás empresas. También depende del efecto riqueza. Por consiguiente, si el mercado de capital es imperfectamente competitivo, en le sentido de que un cambio en la variable de decisión de una empresa afecta al valor de las empresas restantes del mercado, el análisis de la 
formación de la cartera se torna ambiguo a menos que se especifiquen hipótesis sobre preferencia, estimaciones de probabilidades y la estructura del mercado..

\section{Resumen y Conclusiones}

En la primera parte de este documento, se habló sobre el papel de la intermediación financiera en le crecimiento económico. Entonces se destacó que la intermediación en los mercados financieros podría ser una actividad productiva únicamente si el intermediario lograse reducir, de algún modo, los costos de operación de la economía. Generalmente se elimina este tipo de costos en la literatura neoclásica del dinero y las finanzas, de manera que es muy natural que el análisis de la intermediación financiera no haya ocupado un papel preponderante en el trabajo de los economistas monetarios.

Los costos de operación son tan solo una forma en la que se manifiestan las imperfecciones de los mercados de capital. En el mundo real, especialmente en las economías menos desarrolladas, las imperfecciones del mercado se deben en gran parte a factores exógenos, y a la estructura no competitiva de muchos sectores de la economía. En este trabajo se consideraron dos situaciones distintas. Las restricciones exógenas a los créditos, y la ausencia de competencia perfecta en le mercado (en el sentido en que las medidas adoptadas por la empresa o por el individuo pueden afectar los precios del mercado). Se analizó el efecto de estas imperfecciones particulares del mercado sobre las decisiones de la empresa y del consumidor, tanto en el caso de que existe certidumbre perfecta en cuanto a los precios prevalecientes en el mercado y la conducta de otras empresas, como un mundo de incertidumbre. Se encontró que en el caso de la certidumbre, si una mayor inversión de la empresa $i$ tiene un efecto creciente sobre la tasa de interés, el monto que maximiza el valor de la inversión para la empresa es inferior que en el caso de existencia de mercados perfectos. Con respecto a la situación en la que la empresa se enfrenta a límites de crédito, los resultados microeconómicos usuales relacionados con la selección de insumo de la empresa se vuelven ambiguos.

Una situación más realista e interesante es el análisis de los mercados de capital en condiciones de incertidumbre. En la segunda parte del documento se arguye que el tratamiento moderno teórico de selección de cartera bajo condiciones de incertidumbre no es adecuado para analizar mercados de capital imperfectos. En la estructura teórica de este modelo está incorporada la incapacidad para enfrentarse al tipo de incertidumbre "en las transacciones" que nos encontramos cuando los mercados de capital no son perfectos. Sin embargo, est modelo ha sido ampliamente utilizado en la literatura, y 
no fue sino hasta fechas recientes cuando se emprendió una búsqueda de enfoques alternativos (es decir, la literatura reciente sobre la economía de la información).

El modelo de "fijación de precios de activos fijos" (capital asset pricing model) fue empleado para analizar la conducta de inversión de una empresa en dos casos diferentes. Cuando una empresa se enfrenta a restricciones cuantitativas de crédito, asumiendo una conducta de aceptación de precios, se descubre que la aportación de fondos invertidos en la industria sin riesgos es inferior a la del caso normal. No obstante, cuando no se asume la conducta de aceptación de precios, se demuestra que los resultados son ambiguos. En el ejemplo particular que se presenta, la evaluación del precio del riesgo efectuada por la empresa es mayor de lo que sería si se hubiera asumido una conducta de aceptación de precios. Se obtuvo un resultado concreto debido a que se efectuaron varias restricciones sobre preferencias, creencias y la tecnología imperante.

Finalmente, se examinó el efecto sobre la utilidad del consumidor que se produce por decisiones de inversión de empresas bajo condiciones de mercados imperfectos. Se demostró que este efecto depende de la respuesta de todas las empresas cuyas acciones forman parte de la cartera del consumidor, y de efectos "riqueza". El método que se empleó en esta última sección debe de resultar provechoso para analizar situaciones particulares de mercados imperfectos. Como es de esperar, no se obtienen resultados generales al tratar con un mundo que es el "segundo mejor" (second best). Es necesario efectuar un examen particular de cada caso, con el fin de obtener resultados concretos. 


\section{R E F E R E N C I A S}

1. Arrow, K., "Essays in the Theory of Risk Bearing”, Markham, Chicago, 1971.

2. "The Role of securities in the Optimal Allocation of Risk Bearing”, Rev. Econ. Stud., 1964, pp. 91-96.

3. Debreu, G., “Theory of Value”, Yale University Press, New Haven, Conn., 1959.

4. Diamond, P., "The Role of a Stock Market in a General Equilibrium Model with Technological Uncertainty”, Amer. Econ. Rev., 1967, pp. 91-96.

5. Fama, E. And Miller, M., “The Theory of Finance”, Dryden Press, Hinsdale, Ill., 1972

6. Gurley, J. And Shaw, E., "Money in a Theory on finance”, Brookings, Washington, D.C., 1965.

7. Goldsmith, R., "Financial Structure and Development", Yale University Press, New Haven, Conn., 1969.

8. Hicks, J. R., “Capital and Growth”, Oxford University Press, 1965.

9. Hirshleifer, J., “Investment Interest and capital”, Prentice Hall, N.J., 1970.

10. Kapur, B., "Monetary Growth Models of Less Developed Economies", Tesis Doctoral no publicada, Stanford University, 1974

11. Knights, F., $\quad$ "Risk, Uncertainty and Profit”, University of Chicago Press, 1971.

12. Linter, J., "The Valuation of Risky Assets and the Selection of Risky Investments in Stock Portfolios and Capital Budgets, Rev. of Econ and Stat., 1965, pp. 13-37.

13. McKinnon, R., "Money and Capital in Economic Development", Brookings, Washington, D.C., 1973.

14. Mossin, J., “Equilibrium in Capital Asset Market”, Econometrica, 1966, pp. 768-783

15. “Theory of Financial Markets”, Prentince Hall, N.J., 1973. 
16. Nielsen, N., "On the Optimality of Stock Market Allocations", Tesis Doctoral no publicada, Graduate School of Business, Stanford University, 1974.

17. Reynolds, C. and Spellman, L., "Financial Intermediaries and Economic Development as Seen Through the Flow of Funds Accounts”, Food Research Institute Working Paper 1/75, 1975.

18. Sharpe, W., "Capital Asset Price: a Theory of Market Equilibrium Under Conditions of Risk”, Jour. Of Fin., 1964, pp. 425-442.

19. “Portfolio Theory and Capital Markets”, McGraw Hill, N.Y., 1970.

20. Shaw, E., "Financial Deepending in Economic Development", Oxford University Press, 1973.

21. Spellman, L., "Economic Growth and Financial Intermediaries", en McKinnon (ed.) Money and Finance in economic Growth and Development, Essays in Honor of Edward Shaw, Marcel Dekker, N.Y., 1976.

22. Stiglitz, J., "Value Maximization and Alternative Objectives of the Firm", Mimeo, Cowles Foundation, New Haven, Conn., 1972.

23. “Optimality of Stock Market Allocation”, Quart. Jour. of Econ., 1972, pp. 2560.

24. Tobin, J., $\quad$ "Commercial Banks as Creators of Money”, Banking and Monetary Studies, ed. D. Carson, r. Irving, Ill., 1963. 